\title{
Neutral Networks-Based Adaptive Fixed-Time Consensus Tracking Control for Uncertain Multiple AUVs
}

\author{
Lin Zhao \\ College of Automation and Electrical Engineering, Qingdao University, 308 Ningxia Road, Shinan District \\ Qingdao, 266071, China \\ Yingmin Jia \\ The Seventh Research Division and the Center for Information and Control, School of \\ Automation Science and Electrical Engineering, Beihang University (BUAA), 37 Xueyuan Road, Haidian District \\ Beijing, 100191, China \\ Jinpeng Yu \\ College of Automation and Electrical Engineering, Qingdao University, 308 Ningxia Road, Shinan District \\ Qingdao, 266071, China \\ E-mail: zhaolin1585@163.com,ymjia@buaa.edu.cn,yjp1109@hotmail.com \\ http://www.qdu.edu.cn/
}

\begin{abstract}
This paper is concerned with the fixed-time consensus tracking problem for multi-AUV (autonomous underwater vehicle) systems with uncertain parameters and external disturbances. Firstly, a fixed-time terminal sliding mode is proposed, which can avoid the singularity problem. Then, a continuous distributed consensus tracking control law is designed based on Neutral Network approximation technique, which can guarantee the consensus tracking errors converge to the desired regions in fixed time. A simulation example is given to show the effectiveness of proposed methods.
\end{abstract}

Keywords: Multi-AUV systems; Terminal sliding mode; fixed-time stability; Neutral Networks.

\section{Introduction}

Distributed cooperative control of multiple AUVs has been paid to much attention due to its potential applications in oceanographic surveys and deep sea inspections [1]. The distributed cooperative control for multi-AUV systems has been investigated by using the backstepping technique [2] and the adaptive control approach [3]. However, the protocols proposed in them can only guarantee the closed-loop system is asymptotically stable. For the distributed cooperative control, one significant requirement is the fast convergence rate. Compared with the asymptotic control approaches, the finite-time control approaches can not only provide fast convergence rate but also provide higher tracking precision and better disturbance 
rejection ability [4]. Therefore, many finite-time control laws are proposed for various multi-agent systems in the past few years [5]-[7]. However, the settling time can be estimated dependent on the initial conditions of systems in there. In practical applications, we desire that the settling time is estimated independent on the initial conditions of systems. In this paper, we will further investigate the adaptive finite-time consensus tracking problem for multiple AUVs with uncertain dynamics using fixed-time terminal sliding mode.

\section{Systems Description}

This paper considers the networked multiple AUV system with $n$ following AUVs and one virtual leader, and the communications among them are described by a digraph $\bar{G}$. The definitions and descriptions of graph $\bar{G}$ are given in [7] and [9], which is omitted for brevity. Assume that all the following AUVs have fixed attitudes. The translational dynamics of the $i$-th AUV $(i \in V)$ are given as [10]:

$$
\begin{aligned}
& \dot{p}_{i}=R_{i}\left(\Theta_{i}\right) v_{i} \\
& M_{i} \dot{v}_{i}=-D_{i}\left(v_{i}\right) v_{i}-g_{i}\left(\Theta_{i}\right)+\tau_{i}+w_{i}
\end{aligned}
$$

where $p_{i}=\left[x_{i}, y_{i}, z_{i}\right]^{T}, \Theta_{i}=\left[\phi_{i}, \theta_{i}, \psi_{i}\right]^{T}$ denote position and attitude vectors in the inertial reference frame, respectively, $R_{i}\left(\Theta_{i}\right)$ is the kinematic transformation matrix, $v_{i}=\left[u_{i}, v_{i}, \omega_{i}\right]^{T}$ is translational velocity vector in the body-fixed reference frame, $M_{i}$ is the inertia matrix, $D_{i}\left(v_{i}\right)$ is the damping matrix, $g_{i}\left(\Theta_{i}\right)$ is there storing force vector, $\tau_{i} \in \mathfrak{R}^{3}$ is the control force vector, and $w_{i} \in \mathfrak{R}^{3}$ is the disturbance force vector. $M_{i}, R_{i}\left(\Theta_{i}\right)$, $D_{i}\left(v_{i}\right), g_{i}\left(\Theta_{i}\right)$ are defined in [10]. In this paper, we assume that $D_{i}\left(v_{i}\right)$ and $g_{i}\left(\Theta_{i}\right)$ have uncertain parameters. Note that $R_{i} R_{i}^{T}=I$.Denote $p_{d} \in \mathfrak{R}^{3}$ as the state vector of virtual leader and $\dot{p}_{d}, \ddot{p}_{d}$ are all assumed to be smooth, bounded and known functions.

Assumption 1. $\bar{G}$ has a spanning tree, and the leader node is the root node.

\section{Main results}

\subsection{Fixed-time terminal sliding mode (FTTSM)}

Denote

$$
\begin{aligned}
& e_{1 i}=\sum_{j=1}^{n} a_{i j}\left(p_{i}-p_{j}\right)+b_{i}\left(p_{i}-p_{d}\right) \\
& e_{2 i}=\sum_{j=1}^{n} a_{i j}\left(\dot{p}_{i}-\dot{p}_{j}\right)+b_{i}\left(\dot{p}_{i}-\dot{p}_{d}\right)
\end{aligned}
$$

Then, we have $e_{1}=(H \otimes I) \mathrm{E}_{1}, e_{2}=(H \otimes I) \mathrm{E}_{2}$, where $e_{1}=\left[e_{11}^{T}, \ldots, e_{1 n}^{T}\right]^{T}, e_{2}=\left[e_{21}^{T}, \ldots, e_{2 n}^{T}\right]^{T}, \mathrm{E}_{1}=\left[\mathrm{E}_{11}^{T}, \ldots, \mathrm{E}_{1 n}^{T}\right]^{T}$, $\mathrm{E}_{2}=\left[\mathrm{E}_{21}^{T}, \ldots, \mathrm{E}_{2 n}^{T}\right]^{T}, \mathrm{E}_{1 i}=p_{i}-p_{d}, \mathrm{E}_{2 i}=\dot{p}_{i}-\dot{p}_{d}$.

Now, define the FTTSM vector as $s=\left[s_{1}^{T}, \ldots, s_{n}^{T}\right]^{T}$, where $s_{i}=\left[s_{i 1}, s_{i 2}, s_{i 3}\right]^{T} \in \mathfrak{R}^{3}$ is given by

$$
s_{i}=e_{2 i}+\alpha_{i}\left(e_{1 i}\right)
$$

with $\alpha_{i}\left(e_{1 i}\right)=\left[\alpha_{i}\left(e_{1 i 1}\right), \alpha_{i}\left(e_{1 i 2}\right), \alpha_{i}\left(e_{1 i 3}\right)\right]^{T}$,and

$\alpha_{i \chi}\left(e_{1 i \chi}\right)=\left\{\begin{array}{l}\operatorname{sig}\left(\sigma_{1 i} \operatorname{sig}\left(e_{1 i \chi}\right)^{m_{1}}+\sigma_{2 i} \operatorname{sig}\left(e_{1 i \chi}\right)^{n_{1}}\right)^{k_{1}}, \\ \text { if } \bar{s}_{i \chi}=0 \text { or } \bar{s}_{i \chi} \neq 0,\left|e_{1 i \chi}\right|>\phi \\ l_{1 i} e_{1 i \chi}+l_{2 i} \operatorname{sig}\left(e_{1 i \chi}\right)^{2}, \text { if } \bar{s}_{i \chi}=0,\left|e_{1 i \chi}\right| \leq \phi\end{array}\right.$

$\chi=1,2,3, \bar{s}_{i}=\left[\bar{s}_{i 1}, \bar{s}_{i 2}, \bar{s}_{i 3}\right]^{T}, \bar{s}=\operatorname{sig}\left(\sigma_{1 i} \operatorname{sig}\left(e_{1 i \chi}\right)^{m_{1}}+\right.$

$\left.\sigma_{2 i} \operatorname{sig}\left(e_{1 i \chi}\right)^{n_{1}}\right)^{k_{1}}, m_{1}, n_{1}, k_{1} \in \mathfrak{R}^{+}, 0<m_{1} k_{1}<1, n_{1} k_{1}>1$,

$l_{1 i}=\left(2-k_{1}\right)\left(\sigma_{1 i} \phi^{m_{1}-\frac{1}{k_{1}}}+\sigma_{2 i} \phi^{n_{1}-\frac{1}{k_{1}}}\right)^{k_{1}}, l_{2 i}=\left(k_{1}-1\right)$

$\left(\sigma_{1 i} \phi^{m_{1}-\frac{2}{k_{1}}}+\sigma_{2 i} \phi^{n_{1}-\frac{2}{k_{1}}}\right)^{k_{1}}, \phi>0$.

From (3), we can obtain the following equation

$\dot{s}_{i}+s_{i}=\dot{e}_{2 i}+\dot{\alpha}+e_{2 i}+\alpha_{i}$

From the definition of $e_{2 i}$, we further have

$$
\dot{e}_{2 i}=\left(d_{i}+b_{i}\right) \ddot{p}_{i}-\sum_{j=1}^{n} a_{i j} \ddot{p}_{j}-b_{i} \ddot{p}_{d}(6)
$$

and from (1), we can obtain

$$
\ddot{p}_{i}=h_{i}+R_{i}\left(\Theta_{i}\right) M_{i}^{-1} \tau_{i}+R_{i}\left(\Theta_{i}\right) M_{i}^{-1} w_{i}
$$

where $h_{i}=\dot{R}_{i} v_{i}-R_{i} M_{i}^{-1} D_{i} v_{i}-R_{i} M_{i}^{-1} g_{i}$. Assume that $\left\|\left(d_{i}+b_{i}\right) R_{i}\left(\Theta_{i}\right) M_{i}^{-1} w_{i}\right\| \leq \bar{w}_{i}^{*}, \bar{w}_{i}^{*}$ is an unknown constant. Then, substituting (6) into (5) yields

$$
\dot{s}_{i}+s_{i}=\left(d_{i}+b_{i}\right) R_{i}\left(\Theta_{i}\right) M_{i}^{-1} \tau_{i}+\left(d_{i}+b_{i}\right) R_{i}\left(\Theta_{i}\right) M_{i}^{-1} w_{i}+\Phi_{i}
$$

where $\Phi_{i}=-\sum_{j=1}^{n} a_{i j} \ddot{p}_{j}-b_{i} \ddot{p}_{d}+\dot{\alpha}+e_{2 i}+\alpha_{i}+\left(d_{i}+b_{i}\right) h_{i}$. 


\subsection{Control law design}

From the approximation property of RBF Neutral Networks (NNs), we have

$$
\Phi_{i}=W_{i}^{T} \Gamma_{i}\left(Z_{i}\right)+\varsigma_{i}
$$

where $\quad Z_{i}=\left[p_{i}^{T}, \dot{p}_{i}^{T}, p_{j}^{T}, \dot{p}_{j}^{T}, \ddot{p}_{j}^{T}, p_{d}^{T}, \dot{p}_{d}^{T}, \ddot{p}_{d}^{T}\right]^{T} \quad$ and $\left\|\xi_{i}\right\| \leq \xi_{i}^{*}, \xi_{i}^{*}>0$ is a constant. Denote $\hat{\rho}_{i}$ as the estimate of $\rho_{i}=\left\|W_{i}\right\|^{2}$, then the adaptation law is designed as

$$
\dot{\hat{\rho}}_{i}=-2 \kappa_{i} l_{i} \hat{\rho}_{i}+\frac{\kappa_{i}}{2 h_{i}^{2}} s_{i}^{T} s_{i} \Gamma_{i}^{T} \Gamma_{i}
$$

where $\kappa_{i}, l_{i}, h_{i}$ are designed positive constants.

Theorem 1.Suppose that Assumption 1 holds for system (1), then we can choose the control law

$$
\tau_{i}=-\frac{1}{d_{i}+b_{i}} M_{i} R_{i}^{T}\left(\mu_{i 1} \operatorname{sig}\left(s_{i}\right)^{m_{2}}+\mu_{i 2} \operatorname{sig}\left(s_{i}\right)^{n_{2}}+\frac{1}{2 h_{i}^{2}} \hat{\rho}_{i} \Gamma_{i}^{T} \Gamma_{i} s_{i}\right)
$$

where $\mu_{i 1}>0, \mu_{i 2}>0,0<m_{2}<1, n_{2}>1$, such that $s_{i}$ converges into the region

$\left\|s_{i}\right\| \leq \Delta_{s}=\min \left\{2 \mu_{1}^{-\frac{1}{1+m_{2}}}\left(\frac{\Xi}{1-\Xi_{0}}\right)^{\frac{1}{1+m_{2}}}, 2\left(\mu_{2}\left(\frac{1}{2^{\frac{n_{2}+1}{2}}-1}\right)^{n}\right)^{-\frac{1}{1+n_{2}}}\left(\frac{\Xi}{1-\Xi_{0}}\right)^{\frac{1}{1+n_{2}}}\right\}$ in fixed time, the local neighborhood state errors $e_{1 i \chi}$ and $e_{2 i \chi}, \chi=1,2,3$ converge into the regions $\Delta_{e_{1 i}}$ and $\Delta_{e_{2 i}}$ respectively in fixed time, and finally the vectors $\mathrm{E}_{1}$ and $\mathrm{E}_{2}$ converge into regions $\Delta_{\mathrm{E}_{1}}$ and $\Delta_{\mathrm{E}_{2}}$ respectively in fixed time, where $\Delta_{e_{1 i}}=\max \left\{\phi,\left(\frac{\left(\Delta_{s}\right)^{\frac{1}{k_{1}}}}{\sigma_{1 i}}\right)^{\frac{1}{m_{1}}},\left(\frac{\left(\Delta_{s}\right)^{\frac{1}{k_{1}}}}{\sigma_{2 i}}\right)^{\frac{1}{n_{1}}}\right\}$, $\Delta_{e_{2 i}}=\max \left\{\Delta_{s}+l_{1 i} \Delta_{e_{1 i}}+l_{2 i} \Delta_{e_{1 i}}^{2}, \Delta_{s}+\left(\sigma_{i 1} \Delta_{e_{1 i}}^{m_{1}}+\sigma_{2 i} \Delta_{e_{1 i}}^{n_{1}}\right)^{k_{1}}\right\}$, $\mu_{1}=\min \left\{\mu_{1 \text { min }} 2^{\frac{m_{2}+1}{2}}, \varsigma_{\text {min } \frac{m_{2}+1}{2}} 2^{\frac{m_{2}+1}{2}}\right\}, \mu_{1 \text { min }}=\min \left\{\mu_{1 i}\right\}$, $\varsigma_{\min }=\min \left\{\varsigma_{i}\right\}, \mu_{2}=\min \left\{\mu_{2 \min } 2^{\frac{n_{2}+1}{2}}, \varsigma_{\min ^{\frac{n_{2}+1}{2}}} 2^{\frac{n_{2}+1}{2}}\right\}$, $\mu_{2 \min }=\min \left\{\mu_{2 i}\right\}, \varsigma_{i}=\kappa_{i} \frac{\rho_{i}\left(2 o_{i}-1\right)}{2 o_{i}}$, $o_{i}>\frac{1}{2}, 0<\Xi_{0} \leq 1, \Delta_{\mathrm{E}_{1}}=\frac{\sqrt{3 \sum_{i=1}^{n} \Delta_{e_{1 i}}^{2}}}{\sigma_{\min }(H)}, \Delta_{\mathrm{E}_{2}}=\frac{\sqrt{3 \sum_{i=1}^{n} \Delta_{e_{2 i}}^{2}}}{\sigma_{\text {min }}(H)}$.

Proof. Denote $\tilde{\rho}_{i}=\rho_{i}-\hat{\rho}_{i}$, and choose the Lyapunov function as

$$
V=\frac{1}{2} S^{T} S+\frac{1}{2} \sum_{i=1}^{n} \frac{1}{\kappa_{i}} \tilde{\rho}_{i}^{2}
$$

we have

$$
\dot{V} \leq-\sum_{i=1}^{n} s_{i}^{T} s_{i}+\sum_{i=1}^{n} s_{i}^{T}\left(d_{i}+b_{i}\right) R_{i} M_{i}^{-1} \tau_{i}+\sum_{i=1}^{n} \bar{w}_{i}^{*}\left\|s_{i}\right\|+\sum_{i=1}^{n} s_{i}^{T} \Phi_{i}
$$

From

$$
\begin{aligned}
& s_{i}^{T} \Phi_{i} \leq \frac{1}{2 h_{i}^{2}} s_{i}^{T} s_{i}\left\|W_{i}\right\|^{T} \Gamma_{i}^{T} \Gamma_{i}+\frac{1}{2} h_{i}^{2}+\frac{1}{2} s_{i}^{T} s+\frac{1}{2} \xi_{i}^{*, 2} \\
& \bar{w}_{i}^{*}\left\|s_{i}\right\| \leq \frac{1}{2} s_{i}^{T} s+\frac{1}{2} \bar{w}_{i}^{*, 2}
\end{aligned}
$$

Substituting (10), (11), (14) into (13) yields

$$
\begin{aligned}
\dot{V} \leq & -\sum_{i=1}^{n} \sum_{\chi=1}^{3} \mu_{i 1}\left|s_{i \chi}\right|^{m_{2}+1}-\sum_{i=1}^{n} \sum_{\chi=1}^{3} \mu_{i 2}\left|s_{i \chi}\right|^{n_{2}+1}+\sum_{i=1}^{n} 2 \imath_{i} \tilde{\rho}_{i} \hat{\rho}_{i} \\
& +\frac{1}{2} \sum_{i=1}^{n}\left(\bar{w}_{i}^{*, 2}+\xi_{i}^{*, 2}\right)
\end{aligned}
$$

Using the similar proof as in [9], we have

$$
\begin{aligned}
\dot{V} \leq & -\mu_{1} V^{\frac{m_{2}+1}{2}}-\mu_{2}\left(\frac{1}{2^{\frac{n_{2}+1}{2}}-1}\right)^{n} V^{\frac{n_{2}+1}{2}}+\sum_{i=1}^{n}\left(\frac{\varsigma_{i}}{\kappa_{i}} \tilde{\rho}_{i}^{2}\right)^{\frac{m_{2}+1}{2}} \\
& -\sum_{i=1}^{n} \frac{\varsigma_{i}}{\kappa_{i}} \tilde{\rho}_{i}^{2}+\sum_{i=1}^{n} l_{i} o_{i} \rho_{i}^{2}+\frac{1}{2} \sum_{i=1}^{n}\left(\bar{w}_{i}^{*, 2}+\xi_{i}^{*, 2}\right)
\end{aligned}
$$

Suppose that there exists a compact set $\Upsilon$ such that $\Upsilon=\left\{\tilde{\rho}_{i}|| \tilde{\rho}_{i} \mid \leq \Delta\right\}$, then we have

$$
\Xi=\left\{\begin{array}{l}
\sum_{i=1}^{n} l_{i} o_{i} \rho_{i}^{2}+\frac{1}{2} \sum_{i=1}^{n}\left(\bar{w}_{i}^{*, 2}+\xi_{i}^{*, 2}\right), \text { if } \Delta<\min \left\{\sqrt{\frac{\kappa_{i}}{\varsigma_{i}}}\right\} \\
\sum_{i=1}^{n} l_{i} O_{i} \rho_{i}^{2}+\sum_{i=1}^{n}\left(\frac{\varsigma_{i}}{\kappa_{i}} \Delta^{2}\right)^{\frac{m_{2}+1}{2}}-\sum_{i=1}^{n} \frac{\varsigma_{i}}{\kappa_{i}} \Delta^{2}+ \\
\frac{1}{2} \sum_{i=1}^{n}\left(\bar{w}_{i}^{*, 2}+\xi_{i}^{*, 2}\right), \text { if } \Delta \geq \min \left\{\sqrt{\frac{\kappa_{i}}{\varsigma_{i}}}\right\}
\end{array}\right.
$$

From (16) and (17), we can further obtain

$$
\dot{V} \leq-\mu_{1} V^{\frac{m_{2}+1}{2}}-\mu_{2}\left(\frac{1}{2^{\frac{n_{2}+1}{2}}-1}\right)^{n} V^{\frac{n_{2}+1}{2}}+\Xi
$$


It can be seen from Lemma 2 in [8] that the system (12) is practical fixed-time stability. Moreover, $s_{i}$ will converge into the region $\left\|s_{i}\right\| \leq \Delta_{s}$ in fixed settling time.The next proofs are similar with that of [7] and [9], thus are omitted for brevity.

\section{Simulations}

We consider a direct network with three AUVs and a virtual leader, the matrices $\mathrm{L}$ and Bare described as:

$$
L=\left[\begin{array}{ccc}
0 & 0 & 0 \\
-1 & 1 & 0 \\
-1 & 0 & 1
\end{array}\right], B=\left[\begin{array}{ccc}
1 & 0 & 0 \\
0 & 0 & 0 \\
0 & 0 & 0
\end{array}\right]
$$

We assume that all the AUVs have the same structure and the model parameters are $M_{i}=\operatorname{diag}\{175.4,140.8,140.8\}, D_{i}=\left\{120+90\left|u_{i}\right|\right.$, $\left.90+90\left|v_{i}\right|, 150+90\left|\omega_{i}\right|\right\}, \phi_{i}=\pi / 5, \theta_{i}=-\pi / 10, \psi_{i}=\pi / 12$ [10]. The response curves under control law (11) are shown in Fig. 1.Note that the control law(11) can ensure the closed-loop system has desired robustness.

\section{Conclusions}

This paper studied the fixed-time consensus tracking control of Multiple AUVs. AFTTSM based adaptive chattering-free control law was designed, which could guarantee the closed-loop system had desired fixed-time tracking performance.

\section{Acknowledgements}

This work was supported by the National Natural Science Foundation of China (61603204), Natural Science Foundation of Shandong Province (ZR2016FP03), Project funded by China Postdoctoral Science Foundation (2017M612206) and Qingdao Application Basic Research Project (16-5-1-22-jch).

\section{References}

1. T. I. Fossen, Marine control systems: guidance, navigation and control of shipsrigs and underwater vehicles (Trondheim: Marine Cybernetics, 2002).

2. R. S. Cui, S. BGe, V. E. How and Y. S. Choo, Leaderfollower formation control of underactuated autonomous underwater vehicles, Ocean Engineering, 37(1718)(2010) 1491-1502.

3. S. P. Hou and C. C. Cheah, Can a simple control scheme work for a formation control of multiple autonomous underwater vehicles?, IEEE Trans. Contr. Syst. Tech., 19(5) (2011) 1190-1201.
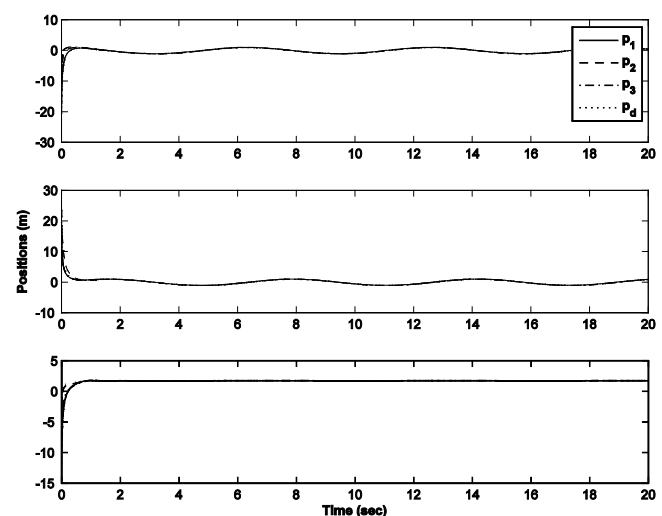

Fig. 1. Response curves of $p_{d}$ and $p_{i}(i=1,2,3)$ under control law (11).

4. L. Zhao and Y. Jia, Finite-time attitude tracking control for a rigid spacecraft using time-varying terminal sliding mode techniques, Int. J. Control, 88(6) (2015) 1150-1162.

5. S. Li, H. Du and X. Lin, Finite-time consensus algorithm for multi-agent systems with double-integrator dynamics, Automatica, 47(8) (2011) 1706-1712.

6. D. Meng,Y. Jia and J. Du, Nonlinear finite-time bipartite consensus protocol for multi-agent systems associated with signed graphs, Int. J. Control, 88(10) (2015) 2074 2085.

7. L. Zhaoand Y. Jia, Decentralized adaptive attitude synchronization control for spacecraft formation using nonsingular fast terminal sliding mode, Nonlinear Dyn., 78(4) (2014) 2779-2794.

8. B. Jiang, Q. Hu and M. I. Friswell, Fixed-time attitude control for rigid spacecraft with actuator saturation and faults, IEEE Trans. Contr. Syst. Tech., 24(5) (2016) 1892-1898.

9. L. Zhao, J. P. Yu, C. Lin and H. S. Yu, Distributed adaptive fixed-time consensus tracking for second-order multi-agent systems using modified terminal sliding mode, Appl. Math. Comput., 312 (2017) 23-35.

10. S. Liand, X. Y. Wang, Finite-time consensus and collision avoidance control algorithms for multiple AUVs, Automatica, 49(11) (2013) 3359-3367. 